\title{
Using the two-source capture-recapture method to estimate the incidence and case ascertainment of congenital rubella syndrome in Australia, 1993-2013
}

\author{
Nicolee Martin, a David Durrheim, ${ }^{b}$ Gulam Khandaker, ${ }^{\text {cdef }}$ Michelle Butler ${ }^{9}$ and Cheryl Jones ${ }^{\text {cdef }}$ \\ Correspondence to Nicolee Martin (email: nicolee.martin@health.gov.au)
}

n 2009, the Technical Advisory Group on Immunization and Vaccine Preventable Diseases in the World Health Organization (WHO) Western Pacific Region endorsed the 2015 targets for accelerating control of rubella and preventing congenital rubella syndrome (CRS). ${ }^{1}$ The global goal outlined in the Global Vaccine Action Plan is for five of six WHO regions, including the Western Pacific Region, to achieve rubella elimination by $2020 .^{2}$

Current evidence suggests that rubella is well controlled and may already be eliminated in Australia. ${ }^{3}$ CRS is now rare, with an average of one case reported annually over the past decade, occurring mostly in infants of unimmunized immigrant mothers. ${ }^{4}$ Rubella and CRS have been nationally notifiable since 1991 with all states and territories notifying confirmed and probable cases to the National Notifiable Diseases Surveillance System (NNDSS). NNDSS is a passive surveillance system, managed by the Commonwealth Department of Health, which collects de-identified data from all Australian states and territories on nationally notifiable diseases. The Australian Paediatric Surveillance Unit (APSU) undertakes active surveillance by child health clinicians who report monthly de-identified clinical laboratory and epidemiological data on a range of conditions, including CRS, since 1993.

To verify rubella and CRS elimination, countries need to ensure that their surveillance systems are sufficiently sensitive to capture almost all cases. This study aims to estimate the incidence of CRS in Australia and the sensitivity of CRS case ascertainment in the NNDSS.

\section{METHODS}

The two-source capture-recapture method $^{5}$ was used to estimate the incidence ofCRS and to evaluate the sensitivity of case ascertainment by the NNDSS. Data on infants born between 1993 and 2013 from NNDSS and APSU were collected and were used to estimate the total number of cases $(N)$ based on the expression $N=a b / c$, where $a$ is the total number of cases ascertained from NNDSS (the primary source), $b$ is the total number ascertained from APSU (the secondary source) and $c$ is the number of cases common to both sources. A modified formula for small numbers ${ }^{5,6}$ was used to estimate CRS incidence between 1993 and 2013:

$$
N=\left[\frac{(a+1)(b+1)}{(c+1)}\right]-1
$$

Estimates were made for the entire 21-year period and additionally stratified by single years and by two time periods, 1993-2003 and 2004-2013, with 95\% confidence intervals $(\mathrm{Cl})$ calculated for the estimated incidence using the formulas: ${ }^{6}$

$$
\begin{aligned}
& \operatorname{var}(N)=\frac{[(a+1)(b+1)(b-c)(a-c)]}{(c+1)^{2}(c+2)} ; \\
& 95 \% \mathrm{Cl}=N \pm Z \sqrt{\operatorname{var}(N)}
\end{aligned}
$$

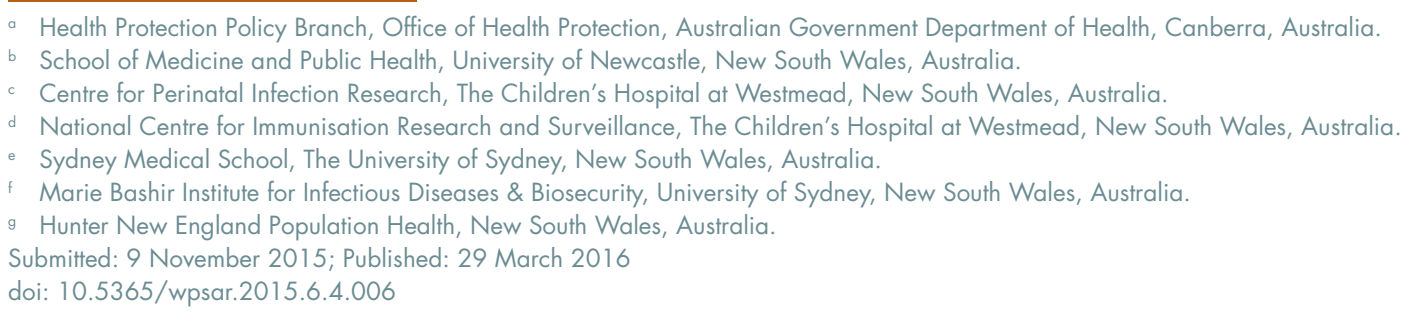


Cases were matched based on date of birth, sex and state or territory of residence. Where cases were matched but their notification dates spanned different years, they were attributed to the earlier of the two notification years. The estimated annual incidence rate (per million live births) was calculated as the sum of reported cases over the sum of the reported live births for the relevant period between 1993 and 2013. ${ }^{7}$ Both confirmed and probable cases, according to the national case definition, ${ }^{8}$ were included in the analysis. Analysis was conducted using Excel (Microsoft Excel 2010, Redmond, WA, USA).

The APSU congenital rubella surveillance study was approved by the Royal Alexandra Hospital for Children (The Children's Hospital at Westmead, New South Wales, Australia) Human Research Ethics Committee. Ethics committee approval was not required for the NNDSS data as the de-identified, aggregated data provided are already available in the public domain.

\section{RESULTS}

Twenty-five cases were identified through the primary source (NNDSS), including 23 confirmed and two probable cases. Thirty-four cases were identified through the secondary source (APSU) and 16 cases were common to both systems for infants born between 1993 and 2013. There were five duplicate notifications identified in the APSU data that were excluded. Three of the 16 cases were mismatched for sex but matched on other parameters. Further investigation found one of the three cases had an incorrectly recorded sex status and another one was further matched by hospital of birth. Both cases were included in the analysis. No further details could be determined for the third case and it was excluded.

An estimate of 56 CRS cases (95\% Cl: 44-68) were expected for the entire 21-year period (19932013), assuming 15 cases common to both sources, representing an overall $45 \%$ case ascertainment for NNDSS. The average birth prevalence in the period 1993 to 2013 was estimated as 9.3 per million live births. When stratifying the data by the two time periods, an average of 18.3 cases per million live births was estimated from 1993 to 2003 compared with 2.2 per million live births from 2004 to 2013.
Case ascertainment by NNDSS was 35\% in 1993-2003 compared with $100 \%$ in $2004-2013$ (Table 1).

\section{DISCUSSION}

This study indicates that CRS incidence in Australia has been low during the 21-year study period with a marked reduction in incidence after 1996 . The significant decrease in incidence is most likely due to introducing a second dose of measles-mumps-rubella vaccine to the National Immunisation Program schedule in 1994, and improved vaccination coverage and population immunity achieved as a result of the Measles Control Campaign in $1998 .{ }^{9}$ Case ascertainment by the NNDSS was poor during the first 11 years of this study but improved substantially to $100 \%$ in all years from 2003 onwards.

The two-source capture-recapture method allows detection of cases by two independent sources to estimate the total number of cases in a given population. This method is based on animal population studies and has been used in epidemiological studies to evaluate completeness of case ascertainment and to estimate the incidence and prevalence of diseases in both human and animal populations. ${ }^{5}$ It has previously proved valuable to validate the sensitivity of acute flaccid paralysis surveillance in Australia. ${ }^{5}$ The use of this method assumes that the primary and secondary sources are independent, all cases have an equal probability of inclusion, cases have been diagnosed accurately and appropriate matching between sources has occurred. ${ }^{10}$ The small number of cases and clinical presentation of CRS combined with a nationally consistent case definition applied by both sources since 2004 assured that most of these assumptions are likely to be met, except that it is difficult to ensure the two sources are completely independent. Nevertheless, they have distinct reporting parthways. While the APSU captures cases reported directly by child health clinicians, the NNDSS is notified of laboratory- or clinically confirmed cases reported to state or territory health departments.

Our analysis reveals CRS incidence in Australia was low and has a marked reduction after 1996. Currently, the NNDSS is sensitive to monitor CRS occurrence and elimination of CRS in Australia. 
Table 1. Estimated annual incidence of CRS and case ascertainment, Australia, 1993-2013

\begin{tabular}{|c|c|c|c|c|c|c|c|}
\hline & $\begin{array}{l}\text { Total from } \\
\text { NNDSS } \\
\text { (primary } \\
\text { source) (a) }\end{array}$ & $\begin{array}{l}\text { Total from } \\
\text { APSU } \\
\text { (secondary } \\
\text { source) }(b)\end{array}$ & $\begin{array}{c}\text { Total } \\
\text { from both } \\
\text { sources }(c)\end{array}$ & $\begin{array}{c}\text { Estimated } \\
\text { total } \\
\text { number of } \\
\text { cases }(N)\end{array}$ & $\begin{array}{c}95 \% \\
\text { confidence } \\
\text { intervals } \\
\text { for } N\end{array}$ & $\begin{array}{c}\text { Estimated } \\
\text { completeness of } \\
\text { ascertainment } \\
(\%)\end{array}$ & $\begin{array}{c}\text { Estimated } \\
\text { incidence of CRS } \\
\text { per million live } \\
\text { births }\end{array}$ \\
\hline Total & 25 & 34 & 15 & 56 & $44-68$ & 45 & 9.3 \\
\hline \multicolumn{8}{|l|}{ Year range } \\
\hline 1993-2003 & 18 & 29 & 10 & 51 & $36-66$ & 35 & 18.3 \\
\hline 2004-2013 & 7 & 5 & 5 & 7 & $7-7$ & 100 & 2.2 \\
\hline \multicolumn{8}{|l|}{ Year } \\
\hline 1993 & 2 & 4 & 0 & 14 & $0-29$ & 14 & 53.8 \\
\hline 1994 & 4 & 6 & 3 & 8 & $6-10$ & 52 & 30.0 \\
\hline 1995 & 1 & 4 & 0 & 9 & 0-18 & 11 & 35.0 \\
\hline 1996 & 5 & 6 & 2 & 13 & $6-20$ & 38 & 37.2 \\
\hline 1997 & 0 & 1 & 0 & 1 & $1-1$ & 0 & 3.9 \\
\hline 1998 & 0 & 1 & 0 & 1 & $1-1$ & 0 & 4.0 \\
\hline 1999 & 1 & 1 & 1 & 1 & $1-1$ & 100 & 4.0 \\
\hline 2000 & 0 & 0 & 0 & 0 & $0-0$ & - & 0.0 \\
\hline 2001 & 0 & 0 & 0 & 0 & $0-0$ & - & 0.0 \\
\hline 2002 & 2 & 3 & 1 & 5 & $2-8$ & 40 & 19.9 \\
\hline 2003 & 3 & 3 & 3 & 3 & $3-3$ & 100 & 11.9 \\
\hline 2004 & 1 & 1 & 1 & 1 & $1-1$ & 100 & 3.9 \\
\hline 2005 & 1 & 0 & 0 & 1 & $1-1$ & 100 & 3.8 \\
\hline 2006 & 0 & 0 & 0 & 0 & $0-0$ & - & 0.0 \\
\hline 2007 & 1 & 0 & 0 & 1 & $1-1$ & 100 & 3.5 \\
\hline 2008 & 1 & 1 & 1 & 1 & $1-1$ & 100 & 3.4 \\
\hline 2009 & 0 & 0 & - & 0 & $0-0$ & - & 0.0 \\
\hline 2010 & 0 & 0 & - & 0 & $0-0$ & - & 0.0 \\
\hline 2011 & 0 & 0 & - & 0 & $0-0$ & - & 0.0 \\
\hline 2012 & 1 & 1 & 1 & 1 & $1-1$ & 100 & 3.2 \\
\hline 2013 & 2 & 2 & 2 & 2 & $2-2$ & 100 & 6.5 \\
\hline
\end{tabular}

APSU, Australian Paediatric Surveillance Unit; CRS, congenital rubella syndrome; and NNDSS, National Notifiable Diseases Surveillance System.

\section{Conflicts of interest}

None declared.

\section{Funding}

The activities of the APSU are funded in part by the Australian Government Department of Health, National Health and Medical Research Council (NHMRC) Practitioner Fellowship No: 1021480 (EE) and an Australian Research Council Linkage Project Grant (LP110200277). Gulam Khandaker is supported by NHMRC Health Practitioners' Early Career Fellowship (1054414) and Cheryl Jones by NHMRC Centres of Research Excellence in Critical Infections (1001021).

\section{References}

1. Meeting Report: Twenty-second meeting of the technical advisory group on immunization and vaccine preventable diseases in the Western Pacific Region, 2013. Manila, World Health Organization Regional Office for the Western Pacific, 2013 (http://www.wpro. who.int/immunization/meetings/2013/tag22_meeting_report.pdf, accessed 8 March 2016).

2. Global Vaccine Action Plan 2011-2020. Geneva, World Health Organization, 2013 (http://www.who.int/immunization/ global vaccine action plan/GVAP doc 2011 2020/en/, accessed 8 March 2016).

3. Song $\mathrm{N}$ et al. Current epidemiology of rubella and congenital rubella syndrome in Australia: progress towards elimination. Vaccine, 2012 , 30:4073-4078. doi:10.1016/j.vaccine.2012.04.025 pmid:22554644

4. Khandaker G, Zurynski Y, Jones C. Surveillance for congenital rubella in Australia since 1993: cases reported between 2004 and 2013. Vaccine, 2014, 32:6746-6751. doi:10.1016/j. vaccine.2014.10.021 pmid:25454858 
5. Whitfield $\mathrm{K}$, Kelly $\mathrm{H}$. Using the two-source capture-recapture method to estimate the incidence of acute flaccid paralysis in Victoria, Australia. Bulletin of the World Health Organization, 2002, 80:846-851. pmid:12481205

6. Cochi SL et al. Congenital rubella syndrome in the United States, 1970-1985. On the verge of elimination. American Journal of Epidemiology, 1989, 129:349-361. pmid:2912045

7. Births, Australia, 1993. Sydney, Australian Bureau of Statistics, 2008 (http://www.abs.gov.au/AUSSTATS/abs@. nsf/second +level + view?ReadForm\&prodno $=3301.0 \&$ viewti tle $=$ Births,$\% 20$ Australia $\sim 1993 \sim$ Previous $\sim 23 / 11 / 1994 \& \&$ tabname $=$ Past $\% 2$ Future $\% 20$ Issues \&prodno $=3301.0 \&$ issue $=1993 \&$ num $=\&$ view $=\&$, accessed 8 March 2016).
8. Australian national notifiable diseases case definitions: Rubella (congenital) case definition. Canberra, Communicable Diseases Network Australia, 2016 (http://www.health.gov.au/internet/main/ publishing.nsf/Content/cda-surveil-nndss-casedefs-cd_conrub. htm, accessed 8 March 2016).

9. Gidding HF et al. Sustained measles elimination in Australia and priorities for long term maintenance. Vaccine, 2007, 25:3574-3580. doi:10.1016/j.vaccine.2007.01.090 pmid: 17300858

10. Hook EB, Regal RR. Capture-recapture methods in epidemiology: methods and limitations. Epidemiologic Reviews, 1995, 17:243264. pmid: 8654510 